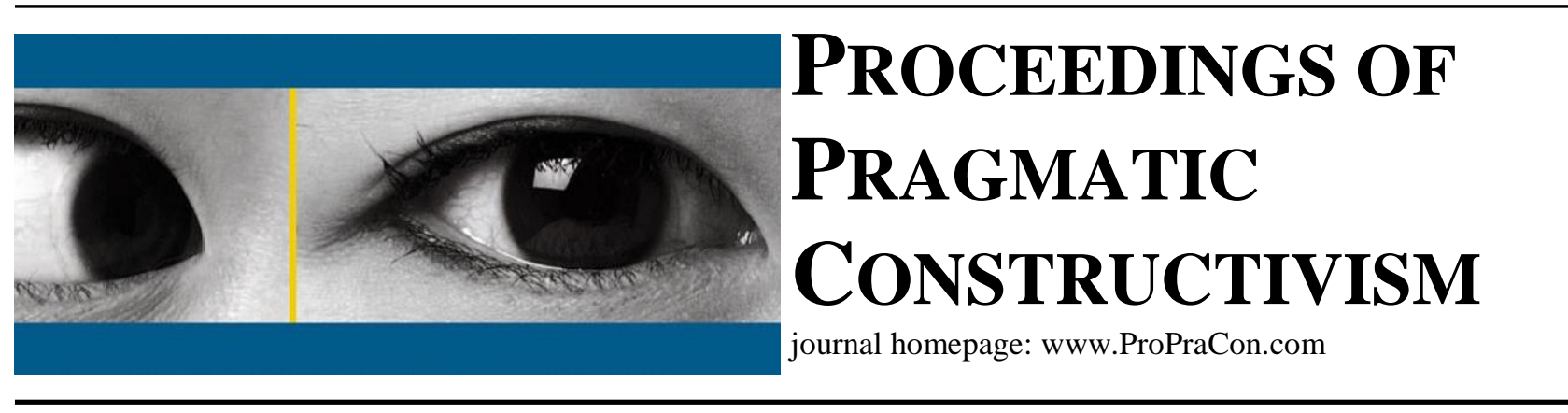

\title{
Editorial: Independent research - reality or illusion?
}

\author{
Morten Jakobsen, \\ Department of Management, Aarhus University \\ Email:mja@mgmt.au.dk \\ Tuomas Korhonen \\ Cost Management Center at the Tampere University \\ Email: tuomas.korhonen@tuni.fi \\ Teemu Laine, \\ Cost Management Center at the Tampere University \\ Email: teemu.laine@tuni.fi
}

\section{Special issue - extended abstracts from the ARC-conference 2019}

We are happy to present volume 9, number 2 of Proceedings of Pragmatic Constructivism. This issue has three extended abstracts that have all been presented at the Actor Reality Construction Conference in Odense October 2019. In each their way, the three contributions are examples of how this field has evolved over the past decade.

The first paper is authored by Lars Bo Henriksen and is titled: New rules of research? - Report from the postmodern university. Lars Bo Henriksen describes two cases from Danish Universities that clearly indicate that independent research is under pressure, perhaps even gone, under the regimes of corporate universities. The two cases are followed by a historical analysis of the conditions for research and running universities. For sure, this is interesting reading, that deserves attention. But perhaps more than reading is required. Obviously, the values of the Humboldtian university are under pressure. But can we as researchers adapt to the change in university management in order to preserve the fundamental values of independent research? Tuomas Korhonen and Teemu Laine were inspired for such reflections based on Lars Bo Henriksen's paper. You can find our reflections on the practices of doing research in close cooperation with practitioners and private and public funders in the section below. If you as reader also want to contribute to the discussion we have opened a blog on the website, where the community can reflect and elaborate upon this important discussion: https://research.tuni.fi/arc/category/blog/

Antonio Leotta, Carmela Rizza, and Daniela Ruggeri present in their paper: The multiple learning behind the process of advising: toward a learning theory of language game, a case of a family business. This family business engages with new actors in order to facilitate a professionalisation of the company. Along with new actors, new concepts and routines evolve. But how does it happen? Through an analysis of the language games that takes place in the organisation, this change process is analysed. The paper is hence an interesting contribution to the ongoing debate in the pragmatic constructivist network concerning language games.

Michael Paulsen contributes to this number with his paper: Understanding the Anthropocene world contemporary difficulties. He argues that we need to develop a new educational language game that is responsive to both our understanding of the world and the world in itself. The paper thereby contributes to the ongoing debate within pragmatic constructivism on language games and its role in reality construction. Michael Paulsen argues that, the fact that we now live in an Anthropocene world requires a shift in our world understanding. This shift must begin with our view on education. Currently educational thinking seems to be rooted in a holocene worldview. This implies that we try to keep on helping new generations to change themselves to function in a world that no longer exists and tie them to a worldview that is highly problematic, reductive and destructive. 


\title{
2 Independent research - possibilities under different governing and funding structures
}

In this issue of Proceedings of Pragmatic Constructivism, the paper by Lars Bo Henriksen has set some thoughts in motion. It is very inspiring and necessitates some self-reflection from the reader's point of view. Such reflection could be beneficial, regardless of the funding of the reader. Below two passages from Henriksen's (2019) text are highlighted:

\begin{abstract}
"When researchers are forced to take on commercial projects and have others outside the university make their problem statements and decide which research projects to take on, then it becomes quite obvious that researchers are caught between a rock and a hard place. They can either take on the commissioned research and generate revenue for the university, but at the same time risk jeopardising research criteria such as validity, reliability and transparency, or they could maintain their professional integrity and defend the classical research criteria, refuse to take on the commissioned research project, and lose their job." (Henriksen, 2019, p. 10)
\end{abstract}

\begin{abstract}
"The question is how researchers should be able to serve both masters - both the commercial as well as the internal research criteria? Neither the rector, nor the minister, can have both - independent research and research for profit. It looks like they expect this to be possible, but they do not have a system capable of supporting these very contrasting expectations." (Henriksen, 2019, p. 10)
\end{abstract}

Working in research projects with external funding, Tuomas Korhonen and Teemu Laine became to think how our situation at the Cost Management Center at Tampere University is similar or different from Lars Bo's paper's thoughts. As brought up by us in the Odense conference, external funding has opened numerous possibilities for cooperation with important actors in interesting research settings. In the situations of change, external funds have also been able to secure us the sort of freedom of research that follows the Humboldtian virtue. With external funding, it has been possible for us to remain relatively autonomous, following our agenda of research and finding new avenues of research together with company participants; with the idea that we apply for such funding that allows us to conduct exactly the type of research we wish, thus adding value to all the actors involved. With external funding, we have been able to select the types of projects we have been willing to initiate or participate in, and as a result, do research as well as we could.

However, we surely identify the fact that our research has not been completely independent, perhaps this is the case in any research setting. We have worked with partners with commercial interests and have tried to add value or support their profit-generation-that is explicitly what some of the governmental sources for innovation funding necessitate: that research must contribute to "international growth" of the private sector, and especially that of the small and medium-sized companies. This governmental (external) funding does not direct us to give certain results. Surely it makes us more interested in aspects that could be funded, based on their funding principles. But, and importantly, this type of funding enables us to study exactly those things that we are interested in: e.g. cost drivers, managerial work, digitalization and interaction in organizations. And, perhaps more importantly, due to the interest of the companies in the particular projects, we have been granted access to in-depth data that would have been unaccessible with other kinds of research funding. This governmental source of funding, though, does not encourage the study of the private sector which is an important issue to realize. Currently we have managed to cover this issue (accidentally or not), e.g. by studying public-private partnerships or the business-to-government market.

Another thought inspired by Henriksen's paper is that (yes) we also want to make the university profit, by not seeking for unprofitable sources of funding. For instance, EU Horizon funding is very tempting for research institutions. EU funding is also very highly competed, and it is always a risk to coordinate a time-consuming application with an international consortium, with possible a less than $10 \%$ acceptance rate. Moreover, the EU-funding can be very much commissioned (indeed it has a strict and specific call for which a proposal is tailored) but the call can be for basic (RIA, research and innovation action) or applied research (IA, innovation action). Still, even though the research project is commissioned, EU does not order results from the researchers. Researhcers still remain to be independent and can still adhere to the principles of the classical university “validity, reliability and transparency” (Lars Bo’s paper). They are even encouraged to do so!

Conclusively, we think Henriksen's (2019) paper opens a very, very interesting debate, that perhaps has numerous tones to it. Hence, we think it is not a case of Humboldtian good, post-Humboldtian bad. It could be more like variety of shades on a continuum with the two mentioned in the extremes. Perhaps this means that a researcher does not necessarily need to make a choice between these two extreme alternatives. The choices of a researcher are not mutually exclusive (perhaps elsewhere than in the extremes of the continuum mentioned)! Importantly, all projects Humboldtian or post-Humboldtian - can succeed or fail in delivering results that we as researchers, the university, and 
the ministers of research can be proud of. Henriksen's (2019) paper is an important reminder that researchers, university managers and funders alike need to be aware of what the integrity of research means.'

\section{References:}

Henriksen, L.B. (2019). New rules of research? - Report from the post-modern university. Proceedings of Pragmatic Constructivism, this issue. 